\title{
Percepção de beleza e cuidados estéticos em mulheres rurais pós-menopausa residentes em um município do interior do Rio Grande do Sul
}

\author{
Marcela Jeani Albarello", Paula Caitano Fontela", Juliana Schneider"**, \\ Evelise Moraes Berlezi ${ }^{* * *}$, Eliane Roseli Winkelmann ${ }^{* * * *}$
}

\section{Resumo}

A diversidade tecnológica na oferta de tratamentos estéticos e a imposição da sociedade pela valorização da beleza podem gerar mudanças de perfil no público feminino que podem ser específicas, considerando-se as características sociodemográficas, econômicas e culturais da população estudada. Este estudo de caráter quantitativo teve como objetivo conhecer a percepção de beleza e os cuidados estéticos em mulheres rurais na pós-menopausa residentes em um município do interior do estado do Rio Grande do Sul. Participaram do estudo, mulheres com idade igual e/ou superior a 65 anos, que faziam parte de um grupo da terceira idade. Foi realizada avaliação dos dados antropométricos e entrevista estruturada com questões sobre a percepção de beleza e a realização de procedimentos estéticos. Foram avaliadas vinte mulheres com média de idade de 69,2 anos, massa corporal e esta- tura média de 73,9 $\mathrm{kg} \mathrm{e} \mathrm{1,62} \mathrm{m} \mathrm{de} \mathrm{altura.}$ Apenas $20 \%$ da amostra havia feito dieta para emagrecimento, a maioria, $45 \%$, considerava a beleza "mais ou menos importante", sendo "muito importante" apenas para $10 \%$. Somente duas delas $(10 \%)$ haviam passado por procedimento cirúrgico, ambas para redução de mama, conforme recomendação médica. Com relação à estética facial e corporal, $40 \%$ e $15 \%$ das mulheres, respectivamente, realizaram algum procedimento para esse fim. A maioria delas nunca obteve conhecimento sobre estética facial e/ ou corporal. Percebe-se que as mudanças na percepção de beleza determinada pela sociedade atual não atinge as mulheres rurais na pós-menopausa residentes em um município do interior do Rio Grande do Sul.

Palavras-chave: Climatério. Envelhecimento da população. Estética. Saúde da mulher.

* Fisioterapeuta, Especialista em Fisioterapia Dermato-Funcional pela Universidade Regional do Noroeste do Estado do Rio Grande do Sul - Unijuí. E-mail: marcelaalbarello@yahoo.com.br

** Fisioterapeuta graduada pela Universidade Regional do Noroeste do Estado do Rio Grande do Sul. Mestranda do Programa de Pós Graduação em Ciências Pneumológicas da Universidade Federal do Rio Grande do Sul - Ufrgs. E-mail: paula fontella@hotmail.com

*** $\quad$ Fisioterapeuta graduada pela Universidade Regional do Noroeste do Estado do Rio Grande do Sul. E-mail: julianaschneider90@yahoo.com.br

***** Fisioterapeuta, doutora em Gerontologia Biomédica pela Pontifícia Universidade Católica do Rio Grande do Sul (PUCRS). Professora do Departamento de Ciências da Vida da Universidade Regional do Noroeste do Estado do Rio Grande do Sul - Unijuí e do mestrado em Atenção Integral à Saúde PPGAIS. Líder do grupo de pesquisa/CNPq Geron - Grupo de Pesquisa em Envelhecimento Humano. E-mail: evelise@unijui. edu.br

****** Doutora em Ciências da Saúde: Cardiologia e Ciências Cardiovasculares pela Universidade Federal do Rio Grande do Sul - Ufrgs. Professora do Departamento de Ciências da Vida da Universidade Regional do Noroeste do Estado do Rio Grande do Sul - Unijuí. Líder do grupo de pesquisa Atenção à Saúde - GPAS e professora do mestrado em Atenção Integral à Saúde PPGAIS. Rua do Comércio, 3000. Bairro Universitário, Departamento de Ciências da Vida (DCVida) da Universidade Regional do Noroeste do Estado do Rio Grande do Sul, CEP: 98.700-000, Ijuí, RS, Brasil. E-mail: elianew@unijui.edu.br

$\rightarrow$ http://dx.doi.org/10.5335/rbceh.v13i1.4969

Recebido em: 25/05/2015. Aceito em: 30/03/2016. 


\section{Introdução}

O conceito de estética, como sinônimo de beleza física, é definido como a qualidade conferida a um corpo por um indivíduo ou por uma determinada sociedade. O binômio beleza-feiura está condicionado a uma questão de gosto, a partir de escolhas subjetivas em relação ao que é visto (CAMARGO et al., 2011). Segundo Gomes e Damazio (2006), estética como atividade profissional tem por finalidade corrigir várias disfunções, além de conservar ou realçar a beleza, por meio de tratamento específico. Uma das disfunções é a obesidade que, além dos seus distúrbios metabólicos, está associada a problemas estéticos, principalmente, ligados à imagem corporal, que corresponde ao retrato mental da própria aparência física (OLIVEIRA; SILVA, 1999; ALMEIDA; OLIVEIRA; VIEIRA, 2008). Um aspecto que pode influenciar mudanças estéticas na mulher é o climatério, período caracterizado por alterações metabólicas e hormonais que, associado ao processo de envelhecimento humano, acarreta várias alterações dermatológicas e estéticas. Landerdahl (2002) afirma que o climatério constitui-se em um processo amplo de transformações de âmbito físico, social, espiritual e emocional, que pode ser mais ou menos extenso para cada sujeito.

Algumas alterações dermatológicas e estéticas são as rugas, as manchas, a flacidez da pele, a gordura localizada, a celulite, entre outras condições provenientes do envelhecimento (COIMBRA; URIBE; OLIVEIRA, 2014). Essas alterações são naturais do processo de envelhecimento cutâneo bem como consequência da constituição genética, de fatores ambientais, da repercussão cutânea do envelhecimento de outros órgãos ou, ainda, podem ser efeitos de doenças da própria pele ou sistêmicas (ACCURSIO, 2001).

Além disso, outros fatores podem agravar o estado físico e emocional dessas mulheres, tais como condição de vida, história reprodutiva, carga de trabalho, hábito alimentar, tendência a infecções, dificuldade de acesso aos serviços de saúde para obtenção de serviços e informações, assim como outros conflitos socioeconômicos, culturais e espirituais associados a esse período da vida e à individualidade (VALENÇA; NASCIMENTO FILHO; GERMANO, 2010).

A imagem corporal é a maneira como o indivíduo vê seu corpo e a si próprio. Os meios de comunicação, a indústria cultural, são os responsáveis por criar desejos e reforçar uma imagem, padronizando corpos (WITT; SCHNEIDER, 2011). Atualmente, os comportamentos mais marcantes do público feminino são o hábito de fazer dietas e de consumir produtos dietéticos, além da busca constante e crescente por procedimentos estéticos, pelo impacto que as alterações corporais propostas podem gerar na imagem corporal (WITT; SCHNEIDER, 2011). No entanto, essa mudança de perfil do público feminino pode ser específica, considerando-se as características sociodemográficas, econômicas e culturais da população estudada.

Tendo em vista a diversidade tecnológica na oferta de tratamentos estéticos, 
a imposição da sociedade pela valorização da beleza e o envelhecimento populacional brasileiro, este estudo teve como objetivo conhecer a relação entre a percepção de beleza e o padrão estético com cuidados estéticos em mulheres rurais na pós-menopausa residentes em um município do interior do estado do Rio Grande do Sul.

\section{Materiais e métodos}

Trata-se de um estudo descritivo de abordagem quantitativa, projetado de acordo com as Diretrizes e Normas Regulamentadoras de Pesquisas Envolvendo Seres Humanos do Conselho Nacional de Saúde Brasileiro e submetido ao Comitê de Ética em Pesquisa da Universidade Regional do Noroeste do Estado do Rio Grande do Sul, sob o Parecer Consubstanciado no 035/2012.

A pesquisa foi realizada em Palmitinho, município que ocupa um território de $144 \mathrm{~km}^{2}$, localizado na Região Noroeste do estado do Rio Grande do Sul. Em 2010, Palmitinho compreendia uma população de 6.920 habitantes. O município foi emancipado em 1966 e tem no setor primário sua principal fonte de renda, distribuída nas atividades de suinocultura, avicultura, gado de leite, cultivo de milho, soja e fumo, além da agricultura familiar, que consiste em atividades na pequena propriedade e que visam à permanência no campo com geração de renda.

A população do estudo foram mulheres rurais que faziam parte do grupo de terceira idade da área urbana do município de Palmitinho, denominado Amigos da Vida, composto por 37 mulheres. Participaram da pesquisa, vinte mulheres que atendiam aos seguintes critérios de inclusão: idade igual ou superior a 65 anos e anuência em participar do estudo.

Foram realizadas entrevistas estruturadas, de forma individualizada, no ambiente em que aconteciam os encontros do grupo. A entrevista consistiu-se, primeiramente, da coleta de dados para a caracterização da amostra. As variáveis de interesse coletadas foram:

a) características sociodemográficas: idade (anos completos), profissão/ ocupação, estado civil (solteira/ casada/viúva);

b) hábitos de vida: prática de exercício físico regular (sim/não) - foi classificada ativa fisicamente, a mulher que relatou realizar qualquer tipo de exercício físico regular com frequência mínima de três vezes por semana -, tipo de exercício físico, tabagismo (sim/não) - considerou-se tabagista a mulher que declarou ser fumante no momento da avaliação, independentemente da quantidade de cigarros consumida -, etilismo ( $\operatorname{sim} /$ não $)$ - considerou-se etilista aquela que referiu consumo excessivo de bebida alcoólica no período da entrevista, em qualquer frequência;

c) presença de doenças e comorbidades: analisadas de acordo com o relato da mulher quando perguntada sobre a presença de doenças cardíacas e/ou respiratórias (sim/ não), hipertensão arterial sistêmica ( $\operatorname{sim} /$ não), diabetes melito ( $\operatorname{sim} /$ não), dislipidemia (sim/não); 
d) hábitos alimentares: obteve-se informações sobre a frequência e os alimentos consumidos durante $o$ café da manhã, lanche da manhã, almoço, lanche da tarde e jantar;

e) medicamentos prescritos: obtidos por meio da consulta à receita médica, apresentada no dia da entrevista.

Após, a entrevista foi direcionada para a captação de informações sobre a percepção da beleza e os procedimentos e cuidados estéticos. As mulheres foram perguntadas quanto à realização de dieta para emagrecimento (com e sem prescrição médica); à importância da beleza (muito importante, importante, mais ou menos importante, não considera importante); ao padrão estético (boa saúde, independente do peso, ser magro, ter peso adequado); à realização de procedimento cirúrgico estético (tipo e motivo); à realização de estética facial e corporal (tipo de tratamento ou procedimento); quando obteve conhecimentos sobre estética facial e corporal (quando jovem, após a menopausa, nunca) e sobre determinados recursos estéticos: desencrust, eletrolifting, eletrolipoforese, luz intensa pulsada, peeling, radiofrequência, ultrassom e vacuometria. As categorias foram constituídas de acordo com as respostas obtidas.

Finalizada a entrevista, foi realizada a avaliação dos dados antropométricos, incluindo a aferição da massa corporal (em quilogramas), utilizando balança digital $\left(\right.$ Toledo $^{\circledR}$ ), e da altura (em metros), em estadiômetro (Toledo ${ }^{\circledR}$ ), segundo técnicas preconizadas. $\mathrm{O}$ índice de massa corporal (IMC) foi calculado pela razão entre o peso corporal e o quadrado da altura: $\mathrm{kg} / \mathrm{m}^{2}$. Foi considerada obesa, a mulher que apresentou IMC $\geq$ $27,0 \mathrm{~kg} / \mathrm{m}^{2}$, conforme proposto por Lipschitz (1994) para indivíduos com idade superior a 60 anos.

A análise estatística foi realizada no programa Statistical Package for Social Sciences (versão 10.0, SPSS, Chicago, Illinois). Os dados foram apresentados em média \pm desvio padrão para variáveis contínuas e frequência absoluta e relativa para variáveis categóricas.

\section{Resultados}

A média de idade das vinte mulheres avaliadas foi de 69,2 anos, foi registrada média de massa corporal e de estatura de $73,9 \mathrm{~kg}$ e $1,62 \mathrm{~m}$, respectivamente, com IMC médio de $28,3 \mathrm{~kg} / \mathrm{m}^{2}$, sendo que $35 \%$ delas foram classificadas como obesas e apenas $50 \%$ relataram praticar exercício físico regular (Tabela 1 ). Nenhuma delas era tabagista ou etilista. Em relação ao estado civil, $65 \%$ eram casadas, $30 \%$ viúvas e $5 \%$ solteiras. Quanto à profissão/ocupação, $40 \%$ eram do lar, $35 \%$ agricultoras, $15 \%$ professoras, $5 \%$ costureiras e $5 \%$ domésticas. A hipertensão arterial sistêmica esteve presente em $70 \%$ delas, as doenças cardíacas em $40 \%$ e a diabetes melito em apenas $5 \%$ das mulheres. Medicamentos de uso regular eram administrados por $80 \%$ delas, com classificação terapêutica anti-hipertensiva, antidiabética, diurética, anti-inflamatória e antiácida. 
Tabela 1 - Caracterização das mulheres rurais na pós-menopausa residentes em um município do Rio Grande do Sul

\begin{tabular}{l|c}
\cline { 2 - 2 } & \multicolumn{1}{c}{$\begin{array}{c}\text { Mulheres } \\
(\mathrm{n}=20)\end{array}$} \\
\hline Idade (anos) & $69,2 \pm 5,0$ \\
Massa corporal $(\mathrm{kg})$ & $73,9 \pm 10,3$ \\
Estatura (m) & $1,62 \pm 0,05$ \\
Índice de massa corporal $\left(\mathrm{kg} / \mathrm{m}^{2}\right)$ & $28,3 \pm 4,1$ \\
Obesidade & $\mathrm{n}(\%)$ \\
Sim & $7(35,0)$ \\
Não & $13(65,0)$ \\
Prática de exercício físico & $10(50,0)$ \\
Tipo de exercício físico & $\mathrm{n}(\%)$ \\
Caminhada & $7(70,0)$ \\
Caminhada + alongamento & $3(30,0)$ \\
\hline
\end{tabular}

Fonte: elaborada pelas autoras com base em dados da pesquisa.

A maioria dessas mulheres $(90 \%)$ con- ria delas (Tabela 2). Com relação à quansumiam cinco refeições diárias, sendo que tidade de água consumida diariamente, pão, arroz, feijão, carne, frutas e saladas 17 (85\%) delas seguem a recomendação eram os alimentos consumidos pela maio- de dois ou mais litros diários.

Tabela 2 - Hábitos alimentares das mulheres rurais pós-menopausa residentes em um município do Rio Grande do Sul

\begin{tabular}{l|r|l|r|l|r}
\hline \multicolumn{1}{c|}{ Turno manhã } & $\begin{array}{c}\text { Consumo } \\
\mathrm{n}(\%)\end{array}$ & Turno tarde & $\begin{array}{c}\text { Consumo } \\
\mathrm{n}(\%)\end{array}$ & Turno noite & $\begin{array}{c}\text { Consumo } \\
\mathrm{n}(\%)\end{array}$ \\
\hline Café da manhã & $19(95)$ & Almoço & $20(100)$ & Jantar & $16(80)$ \\
Café & $11(58)$ & Feijão & $19(95)$ & Café & $4(25)$ \\
Pão & $17(89)$ & Arroz & $20(100)$ & Pão & $2(11)$ \\
Queijo & $6(32)$ & Carne & $20(100)$ & Bolacha & $3(17)$ \\
Chimia & $3(16)$ & Batata & $6(30)$ & Massa & $3(17)$ \\
Bolacha & $5(26)$ & Mandioca & $5(25)$ & & \\
Leite & $11(58)$ & Massa & $9(45)$ & & \\
Presunto & $1(5,3)$ & Salada & $17(85)$ & & \\
Margarina & $3(16)$ & Ovos & $1(5)$ & & \\
Salame & $2(10,5)$ & Legumes & $1(5)$ & & \\
Mortadela & $1(5,3)$ & Lanche da tarde & $18(90)$ & & \\
Frutas & $8(42)$ & Frutas & $12(67)$ & & \\
Aveia & $2(10,5)$ & Bolacha & $13(72)$ & & \\
Lanche da manhã & $14(70)$ & Chá & $4(22)$ & & \\
Bolacha & $5(36)$ & Doces & $1(5,5)$ & & \\
Frutas & $11(79)$ & Bolo & $10(55,5)$ & & \\
\hline
\end{tabular}

Fonte: elaborada pelas autoras com base em dados da pesquisa. 
Apenas 20\% da amostra já havia feito dieta para emagrecimento, dessas, $75 \%$ foi por prescrição médica. A maioria (45\%) considera a beleza "mais ou menos importante", sendo "muito importante" apenas para duas mulheres (10\%). Apenas 1 mulher (5\%) equiparava o padrão estético com o fato de "ser magra". Somente duas delas (10\%) passaram por procedimento cirúrgico, ambas para redução de mama, conforme recomendação médica (Tabela 3).

Tabela 3 - Realização de dieta e de procedimento cirúrgico estético e importância da beleza e padrão estético em mulheres rurais na pós-menopausa residentes em um município do Rio Grande do Sul

\begin{tabular}{l|r} 
& $\begin{array}{r}\text { Mulheres } \\
(\mathrm{n}=20)\end{array}$ \\
\hline Realização de dieta para & $4(20)$ \\
emagrecimento & $3(75)$ \\
Com prescrição médica & $1(25)$ \\
SEM prescrição médica & \\
Importância da beleza & $2(10)$ \\
Muito importante & $8(40)$ \\
Importante & $9(45)$ \\
Mais ou menos importante & $1(5)$ \\
Não considera importante & - \\
Padrão estético & $2(100)$ \\
Boa saúde independente do peso & $1(5)$ \\
Ser magro & - \\
Peso adequado & - \\
Realização de procedimento cirúrgico \\
estético
\end{tabular}

Com relação à estética facial, $40 \%$ das mulheres entrevistadas realizaram procedimento para esse fim, sendo $30 \%$ para limpeza de pele. Esses resultados são menores ainda, quando perguntadas a respeito da estética corporal, pois apenas três mulheres (15\%) realizaram algum procedimento com essa finalidade: gordura localizada e celulite. Nenhuma delas realizou tratamento para estrias, flacidez muscular/pele e discromias e, após a menopausa, não realizaram qualquer procedimento estético. A maioria dessas mulheres nunca obteve conhecimento sobre estética facial $(60 \%)$ e/ou corporal (85\%), sendo observado maior conhecimento e interesse sobre estética facial. Além disso, a maioria delas obteve tais conhecimentos após a menopausa (30\%), demonstrando que para essas mulheres a maior preocupação é com a beleza facial, momento em que as alterações estéticas provenientes do processo do envelhecimento estão mais evidentes. Para mulheres que manifestaram ter conhecimento sobre estética corporal, esses foram obtidos quando elas eram jovens, mostrando que, após a menopausa, essas mulheres apresentam desinteresse e despreocupação com a estética corporal (Tabela 4). 
Tabela 4 - Realização e conhecimento de estética facial e corporal em mulheres rurais na pós-menopausa residentes em um município do Rio Grande do Sul

\begin{tabular}{l|r}
\cline { 2 - 2 } & \multicolumn{1}{|c}{$\begin{array}{c}\text { Mulheres } \\
(\mathrm{n}=20)\end{array}$} \\
\hline Realização de estética facial & $8(40)$ \\
Quando obteve conhecimento sobre & \\
estética facial & $2(10)$ \\
Quando jovem & $6(30)$ \\
Após a menopausa & $12(60)$ \\
Nunca & $1(5)$ \\
Realizou tratamento para rugas & $6(30)$ \\
Realizou limpeza de pele & $1(5)$ \\
Realizou procedimento para rejuve- \\
nescimento \\
Realização de estética corporal & $3(15)$ \\
Quando obteve conhecimento sobre \\
estética corporal
\end{tabular}

Fonte: elaborada pelas autoras com base em dados da pesquisa.

Quando perguntadas sobre recursos estéticos, três mulheres (15\%) conheciam a luz intensa pulsada, uma (5\%) a radiofrequência, o desencrust e a eletrolipoforese, $2(10 \%)$ o eletrolifting, $8(40 \%)$ a vacuometria, 17 (85\%) o ultrassom e 15 (75\%) o peeling.

\section{Discussão}

Embora a pós-menopausa seja um período natural, que ocorre na vida de uma mulher, a literatura científica em todo o mundo relata muitos problemas de ordem fisiológica, emocional, social e cultural nesse período (LANDERDAHL, 2002; DE LORENZI et al., 2005; DENNERSTEIN; LEHERT; GUTHRIE, 2002; BAULD; BROWN, 2009; HUNTER; RENDALL, 2007). De acordo com levantamento de dados pelos pesquisadores, este é o primeiro estudo sobre a relação entre percepção de beleza e cuidados estéticos em mulheres rurais pós-menopausa. Encontrou-se somente um estudo que investigou mulheres da área rural - porém, diferentemente das mulheres incluídas no presente estudo, eram mulheres com idade inferior a 50 anos - cujo objetivo era avaliar a preocupação com o corpo e as mudanças nas práticas alimentares a fim de obter o corpo desejado (PÉREZ GIL-ROMO; VEGA GARCÍA; ROMERO-JUÁREZ, 2007).

A idade parece não ser um fator determinante para definir o significado da beleza para a mulher e interferir nos cuidados com o seu corpo. Um estudo transversal, realizado em uma população urbana alemã com 102 mulheres de idades entre 50 e 82 anos evidenciou que a percepção do corpo é tão sensível em mulheres mais velhas, quanto em mulheres de meia idade, assim como a sexualidade é importante para ambas, independentemente da faixa etária (VON GRUCHALLA et al., 2003).

As mulheres do presente estudo contavam com informações sobre tecnologia e conhecimento sobre estética da década de 1940/1950, quando o padrão de beleza era outro, e não havia recursos, sendo que os existentes eram pouco divulgados. Isso foi percebido quando a minoria da amos- 
tra relatou ter conhecimento sobre determinados recursos estéticos. Durante muito tempo, na história da humanidade, o ganho de peso, bem como o acúmulo de gordura, era visto como sinal de saúde e prosperidade (WORLD HEALTH ORGANIZATION, 1998). Pensando em termos de corpo, o referencial de beleza e saúde feminina era o modelo do corpo da boa mãe (SILVA; GODÓI, 2009). Acreditava-se que a feminilidade estaria refletida em características corporais propícias à maternidade: seios volumosos, corpo arredondado, ancas desenvolvidas (CARVALHO; PICCININI, 2008).

Entretanto, ao longo dos séculos, os padrões de beleza feminina mudaram e hoje a sociedade contemporânea busca incessantemente o "corpo perfeito" (MORGAN; AZEVEDO, 1998), esse tema é de interesse geral na atualidade, pois cada vez mais, as pessoas têm a sua identidade ligada a essa imagem (SAVOIA, 2003), porém parece não fazer parte da vida das mulheres estudadas. O padrão estético muda de tempos em tempos, de sociedade para sociedade, pois é uma construção cultural, social e histórica, portanto, também podem variar em diferentes grupos sociais (SILVA; GODÓI, 2009). Esses fatores, certamente, determinaram a percepção das mulheres estudadas sobre a importância da beleza e do padrão estético, pois ser uma mulher bela, urbana, nos anos 2000/2010, é diferente de ser uma mulher bela, rural, nas décadas de 1960/1970, cujo padrão mais roliço indicava sinônimo de saúde.

Mesmo na atualidade, com inúmeros recursos e técnicas cada vez mais eficazes no tratamento estético facial e corporal, na amostra avaliada, os procedimentos que foram realizados podem ser considerados simples, ou seja, mesmo com a inovação e a socialização dos procedimentos pelos meios de comunicação e pelas novas exigências no padrão de beleza, essas mulheres se mostraram muito cautelosas na aceitação desses modelos e procedimentos. Também, o que pode influenciar no não conhecimento das novas tecnologias por essas mulheres é $o$ fato que a cidade/região onde residem é um local distante dos centros mais avançados e atualizados em relação a essas tecnologias.

Uma das principais mudanças causadas pelo envelhecimento é a perda da elasticidade da pele, isto ocorre devido à redução de fibras elásticas, o que causa rugas e flacidez (GUIRRO; GUIRRO, 2004). Muitos recursos, como cosméticos e cirurgias, são utilizados para o tratamento dessas alterações. $\mathrm{Na}$ amostra estudada, nenhum desses recursos foi utilizado, provavelmente, pela percepção que a maioria das mulheres tem de que a beleza é mais ou menos importante, visto que na concepção dessas mulheres, beleza e padrão estético é ter boa saúde. Estimando que essa concepção é resultado de uma construção histórica, cultural e social, deve-se considerar, além da idade, os modos de vida e as condições econômicas da amostra. As mulheres pesquisadas eram da área rural e tinham como principal atividade ocupacional a dedicação ao lar e à agricultura, principal fonte de renda do município. 
A evolução dos procedimentos estéticos deve-se a um novo contexto social, cultural e tecnológico que a sociedade está impondo. Dessa forma, recursos, como a luz intensa pulsada, a radiofrequência, o desencrust, a eletrolipoforese, o eletrolifting, a vacuometria, o peeling entre outros procedimentos, atingem, em sua grande parte, mulheres em vida social ativa, com determinada independência econômica, o que não caracteriza a população estudada.

O presente estudo evidenciou uma porcentagem considerável de mulheres obesas. Tal achado pode ser decorrente de hábitos alimentares inadequados e baixa adesão à prática regular de exercício físico constatada neste estudo, o que gera um balanço energético positivo que resulta em ganho de massa corporal e, consequentemente, em obesidade. Esse estilo de vida com hábitos pouco saudáveis pode ser resultante das características socioculturais da amostra estudada, mulheres pós-menopausa da área rural de um município do interior do estado pouco afeitas à beleza e à estética. Um estudo qualitativo realizado em 2007, com 37 mulheres da área rural da comunidade de Huatecalco, no México, mostrou que para elas a magreza era equiparada à beleza, algumas delas tinham preocupação com seu corpo e, também, que a publicidade em torno da perda de peso estava modificando algumas das práticas alimentares das mulheres investigadas, fenômeno não encontrado na presente amostra, mesmo tratando-se de mulheres em situação socioeconômica semelhante, porém mais jovens (PÉREZ GIL-ROMO; VEGA GARCÍA; ROMERO-JUÁREZ, 2007).

Além desses fatores, a obesidade nas mulheres investigadas pode ser resultante do processo de envelhecimento, pois as modificações na composição e distribuição do tecido adiposo são resultantes de alterações no perfil metabólico, o que favorece não somente o aumento ponderal, como também a progressão de eventuais processos ateroscleróticos (BORTOLUZZI, 2010). Essas modificações, muitas, além de considerações estéticas, estão, reconhecidamente, implicadas na gênese e progressão da doença cardiovascular, que constitui a principal causa de mortalidade, principalmente em indivíduos idosos, tanto do sexo masculino quanto do feminino (JOUSILAHTI et al., 1999).

A falta de atividade física é apontada como causa de inúmeros danos à saúde e tem como consequência direta ou indireta o aparecimento de doenças (DE VITTA, 2001). Esse fato associado à má nutrição aumenta o risco de desenvolvimento de enfermidades frequentes na terceira idade, como hipertensão arterial sistêmica (FAGARD, 2005). Além disso, o aumento da pressão arterial sistêmica, em mulheres, relacionado à idade é, possivelmente, devido ao declínio da produção de estrógenos no climatério e menopausa (PLAVNIK; ROSA; KOHLMANN, 2007), o que pode explicar a alta prevalência de hipertensão arterial nas mulheres estudadas.

$\mathrm{O}$ presente estudo apresenta algumas limitações. A principal limitação é, sem dúvida, o tamanho da amostra. 
Além disso, a amostra provém de um único local com características específicas. Portanto, os resultados não podem ser generalizados. Entretanto, o presente estudo contribui com a divulgação de resultados singulares sobre a percepção de beleza e cuidados estéticos em mulheres rurais pós-menopausa, população pouco explorada em estudos sobre temas relacionados.

Outras limitações referem-se ao fato de que informações sobre uso e tipo de terapia de reposição hormonal e prevalência de sintomas climatéricos não foram sistematicamente avaliadas. Portanto, os dados encontrados podem estar relacionados a esses fatores, e não simplesmente às características da população estudada, pois a terapia de reposição hormonal e os sintomas climatéricos podem influenciar nos aspectos psicológico e emocional da mulher que, consequentemente, podem influenciar em suas percepções e cuidados (BIEN et al., 2015; FAUCONNIER et al., 2000; TAKEDA et al., 2012).

Foi demonstrado em um estudo recente que a intensidade dos sintomas climatéricos afeta significativamente a vida e as atividades da mulher. As mulheres que apresentavam sintomas climatéricos moderados ou graves tinham sentimentos mais fortes de insuficiência, mais sentimentos de oportunidades disponíveis e de perda da sua juventude e beleza quando comparadas àquelas que apresentavam nenhum sintoma ou sintomas leves (BIEN et al., 2015).
Estudos mostram a associação entre terapia de reposição hormonal e práticas e cuidados de beleza. Em estudo realizado na França com 561 mulheres pós-menopausa, das quais 72,9\% estavam usando terapia de reposição hormonal durante mais de um ano e $27,1 \%$ tinham usado por menos de três meses por pelo menos uma vez, a prática e o cuidado de beleza foi independentemente associado com o uso de terapia de reposição hormonal (FAUCONNIER et al., 2000). Em outro estudo, o nível de atenção aos cuidados de beleza desempenhou papel na escolha da formulação da terapia de reposição hormonal (TAKEDA et al., 2012).

\section{Conclusão}

Percebe-se que as mudanças na percepção de beleza determinada pela sociedade atual não atinge as mulheres rurais pós-menopausa residentes em município do interior do estado do Rio Grande do Sul, participantes deste estudo, que não consideram beleza um fator muito importante, não possuem um bom conhecimento sobre estética, não buscaram se atualizar sobre as novas tecnologias na área e também são cautelosas na aceitação dos recursos e tratamentos estéticos atuais. Dessa forma, o significado da beleza para a mulher, não ocorre de uma única perspectiva, mas como resultado de uma inter-relação de experiências, costumes e mitos sociais e pessoais. 


\section{Beauty perception and beauty care in postmenopausal rural women living in a country city from Rio Grande do Sul State}

\section{Abstract}

The technological diversity in offering aesthetical treatments and the society imposition by beauty appreciation can generate female public profile changes that can be specific, taking into account the sociodemographic characteristics, economic and cultural of the population studied. This study of quantitative character had as purpose to know the beauty perception and the aesthetic care in postmenopausal rural women living in a country city from Rio Grande do Sul State. Women who participated this study, with age the same and/or higher to 65 years-old, which were part of third age group. Was held anthropometric data evaluation and structured interview having questions approaching the beauty perception and the aesthetic procedures holding. Were evaluated 20 women with average age of 69,2 years old, body mass and average height of $73,9 \mathrm{~kg}$ and 1,62 m, respectively. Only $20 \%$ of sample held slimming diet, the most, $45 \%$, considered the beauty "more or less important", being "very important" only for $10 \%$. Only two of them $(10 \%)$ held surgical procedure, being both for breast reduction according to medical recommendation. With regard to facial and physical aesthetics, $40 \%$ and $15 \%$ of women, respectively, held some procedure for this reasons. The most of them never got knowledge about facial and/or physical aesthetics. It understands that the beauty perception changes determined by current society not achieve the postmenopausal rural women living in a country city from Rio Grande do Sul State.

Keywords: Climacteric. Demographic aging. Esthetics. Women's health.

\section{Referências}

ACCURSIO, Célia Sampaio Costa. Alterações de pele na terceira idade. Revista Brasileira de Medicina, São Paulo, v. 58, n. 9, p. 646-650, 2001.

ALMEIDA, Clarissa Mendonça Erdmann; OLIVEIRA, Maria Rita Marques de; VIEIRA, Carla Maria. A relação entre a imagem corporal e obesidade em usuárias de unidades de saúde da família. Simbio-Logias, Botucatu, v. 1 , n. 1, p. 111-21, 2008.

BAULD, Rosie; BROWN, Rhonda F. Stress, psychological distress, psychological factors, menopause symptoms and physical health in women. Journal: Maturitas, v. 62 , n. 2 , p. $160-165,2009$.

BIEN, Agnieszka et al. The influence of climacteric symptoms on women's lives and activities. International Journal of Environmental Research and Public Health, v. 12, n. 4, p. 3.835-3.846, 2015.

BORTOLUZZI, Scheila Bittencourt de Oliveira. Avaliação do consumo alimentar com base na dieta do mediterrâneo em pacientes portadores de doença arterial coronariana de um hospital do município de Criciúma. 39 p. Trabalho de conclusão de curso (Bacharelado em Nutrição) - Universidade do Extremo Sul Catarinense, Criciúma, 2010.

CAMARGO, Brigido Vizeu et al. Representações sociais do corpo: estética e saúde. Temas em Psicologia, Ribeirão Preto, v. 19, n. 1, p. 257-268, 2011.

CARVALHO, Fernanda Torres de; PICCININI, Cesar Augusto. Aspectos históricos do feminino e do maternal e a infecção pelo HIV em mulheres. Ciência \& Saúde Coletiva, Rio de Janeiro, v. 13, n. 6, p. 17-31, 2008.

COIMBRA, Daniel Dal'Asta; URIBE, Natalia Caballero; OLIVEIRA, Betina Stefanello. "Quadralização facial" no processo do envelhecimento. Surgical and Cosmetic Dermatology, Rio de Janeiro, v. 6, n. 1, p. 65-71, 2014. 
DE LORENZI, Dino Roberto Soares et al. Fatores indicadores da sintomatologia climatérica. Revista Brasileira de Ginecologia e Obstetrícia, Rio de Janeiro, v. 27, n. 1, p. 12-19, 2005.

DE VITTA, Alberto. Bem-estar físico e saúde percebida: um estudo comparativo entre homens e mulheres, adultos e idosos, sedentários e ativos. Tese (Doutorado em Educação) - Universidade Estadual de Campinas, Campinas, 2001.

DENNERSTEIN, Lorraine; LEHERT, Philippe; GUTHRIE, Janet. The effects of the menopausal transition and biopsychossocial factors on well-being. Archives of Women's Mental Health, v. 5, n. 1, p. 15-22, 2002.

FAGARD, Robert H. Physical activity, physical fitness and the incidence of hypertension. Journal of Hypertension, v. 23, n. 2, p. 265-267, 2005.

FAUCONNIER, Arnaud et al. Use of hormone replacement therapy: women's representations of menopause and beauty care practices. Journal: Maturitas, v. 35, n. 3, p. 215-228, 2000.

GOMES, Roseline K.; DAMAZIO, Marlene Gabriel. Cosmetologia: descomplicando os princípios ativos. São Paulo: LMP, 2006.

GUIRRO, Elaine; GUIRRO, Rinaldo. Fisioterapia Dermato-Funcional. Barueri: Manole, 2004.

HUNTER, Myra; RENDALL, Melanie. Bio-psycho-socio-cultural perspectives on menopause. Best Practice and Research Clinical Obstetrics and Gynaecology, v. 21, n. 2, p. 261-274, 2007.

JOUSILAHTI, Pekka. et al. Sex, age, cardiovascular risk factors, and coronary heart disease: a prospective follow-up study of 14 786 middle-aged men and women in Finland. Circulation, v. 99, n. 9, p. 1.165-1.172, 1999.

LANDERDAHL, Maria Celeste. Mulher climatérica: uma abordagem necessária ao nível da atenção básica. Nursing, São Paulo, v. 5 , n. 47 , p. 20-25, 2002.

LIPSCHITZ, David A. Screening for nutritional status in the elderly. Prim Care, v. 21, n. 1, p. 55-67, 1994.

MORGAN, Christina Marcondes; AZEVEDO, Angélica M. Claudino. Aspectos sócio-culturais dos transtornos alimentares. Psychiatry On-Line Brazil, São Paulo, v. 3, n. 2, 1998.

OLIVEIRA, João Joaquim; SILVA, Sandra Regina A. S. Obesidade e coração: a forma e localização vão muito além de um mero problema estético. Jornal Brasileiro de $\mathrm{Me}$ dicina, v. 77, n. 5-6, p. 84-94, 1999.

PÉREZ GIL-ROMO, Sara Elena; VEGA GARCÍA, Luz Amaranta; ROMERO-JUÁREZ, Gabriela. Prácticas alimentarias de mujeres rurales: ¿una nueva percepción del cuerpo? Salud Pública de México, v. 49, n. 1, p. 52-62, 2007.

PLAVNIK, Frida Liane; ROSA, Eduardo Cantoni; KOHLMANN JR, Osvaldo. Hipertensão arterial sistêmica na mulher. Revista Brasileira de Medicina, São Paulo, v. 64, n. 3, p. 88-94, 2007.

SAVOIA, Mariangela Gentil. A imagem corporal. Revista Brasileira de Psiquiatria, São Paulo, v. 25, n. 2, p. 126-126, 2003.

SILVA, Runy Cristina da; GODÓI, Marcos Roberto. O desejo e os riscos de um corpo 'arquitetado': corpos femininos e cirurgias plásticas em Cuiabá-MT, Brasil. Efdeportes. com/Revista Digital, v. 14, n. 136, 2009.

TAKEDA, Takashi et al. Estrogen formulations and beauty care practices in Japanese women. International Journal of Women's Health, v. 4, p. 19-24, 2012.

VALENÇA, Cecília Nogueira; NASCIMENTO FILHO, José Medeiros do; GERMANO, Raimunda Medeiros. Mulher no climatério: reflexões sobre desejo sexual, beleza e feminilidade. Saúde e Sociedade, São Paulo, v. 19, n. 2, p. 273-285, 2010. 
VON GRUCHALLA, B. et al. Peri- und Postmenopause: Körperlichkeit, Sexualität und Selbstbild der älteren Frau. Zentralblatt für Gynäkologie, Stuttgart, v. 125, n. 6, p. 202-208, 2003.

WITT, Juliana da Silva Gonçalvez Zanini; SCHNEIDER, Aline Petter. Nutrição estética: valorização do corpo e da beleza através do cuidado nutricional. Ciência e Saúde Coletiva, Rio de Janeiro, v. 16, n. 9, p. 3.909-3.916, 2011.

WORLD HEALTH ORGANIZATION. Obesity status: preventing and managing the global epidemic. Report of a WHO consultation on obesity. Geneva: WHO, 1998. 